06

\title{
Нелинейные поверхностные волны в симметричной трехслойной структуре из оптических сред с различными механизмами формирования нелинейного отклика
}

\author{
(C) C.E. Савотченко \\ Белгородский государственный технологический университет им. В.Г. Шухова, \\ 308012 Белгород, Россия \\ e-mail: savotchenkose@mail.ru
}

Поступила в редакцию 26.04.2019 г.

В окончательной редакции 24.11.2019 г.

Принята к публикации 26.11.2019 г.

Рассмотрено распространение поверхностных ТМ-волн в трехслойной структуре, представляющей собой пластину конечной толщины из кристалла с фокусирующей нелинейностью керровского вида, зажатую между одноосными фоторефрактивными кристаллами с диффузионным механизмом формирования нелинейности. Вдоль границ раздела слоев могут распространяться нелинейные поверхностные волны, различающиеся характером затухания. Амплитуда волны одного вида убывают при удалении от границ раздела без осцилляций в глубину внешних слоев из фоторефрактивных кристаллов, а другого - убывают с осцилляциями. Профили волн могут иметь две формы симметрии относительно центра трехслойной структуры: симметричные и антисимметричные. Волны, амплитуда которых убывает в глубину фоторефрактивных кристаллов как с осцилляциями, так и без них, с симметричным распределением существуют двух типов, а с антисимметричным - одного. В явном аналитическом виде найдены зависимости константы распространения от характеристик слоистой структуры для длинноволнового режима распространения поверхностных волн, а также указаны условия их существования.

Ключевые слова: слоистая среда, трехслойная структура, фоторефрактивный кристалл, нелинейная среда, керровская нелинейность, нелинейные поверхностные волны.

DOI: $10.21883 /$ OS.2020.03.49064.196-19

\section{Введение}

К настоящему времени существует много работ, в том числе и теоретических, посвященных исследованиям свойств поверхностных волн в оптических слоистых средах [1-3]. Широкое распространение среди исследователей получили трехслойные структуры [4] в связи с их применением в интерферометрах [5].

Особое значение имеют нелинейные поверхностные волны в силу целого ряда их специфических свойств $[6,7]$. Такие волны распространяются в средах, в которых возможно формирование нелинейного отклика, возникающего вследствие перераспределения плотности зарядов, внешнего поля и других факторов [8].

К наиболее распространенным нелинейным оптическим средам относятся кристаллы с эффектами Поккельса $\left(\mathrm{KH}_{2} \mathrm{PO}_{4}, \mathrm{NH}_{4} \mathrm{H}_{2} \mathrm{PO}_{4}, \mathrm{LiNbO}_{3}\right)$ и Kерра $\left(\mathrm{BaTiO}_{3}\right.$, $\mathrm{SrTiO}_{3}$ ), в которых наблюдаются соответственно линейная и квадратичная зависимости показателя преломления от напряженности электрического поля (или компонент тензора диэлектрической проницаемости в анизотропных кристаллах) [9]. Кроме того, в различных оптоэлектронных устройствах стали активно использоваться кристаллы с фоторефрактивным эффектом $\left(\mathrm{LiTaO}_{3}, \mathrm{BaTiO}_{3}, \mathrm{KNbO}_{3}, \mathrm{Fe}: \mathrm{LiNbO}_{3}\right.$, семейство силленитов: $\mathrm{Bi}_{12} \mathrm{SiO}_{20}, \mathrm{Bi}_{12} \mathrm{TiO}_{20}, \mathrm{Bi}_{12} \mathrm{GeO}_{20}$, в том числе с легированием примесями) [10-12].
Механизмы возбуждения поверхностных волн в таких кристаллах играют важную роль в оптоэлектронике, что и обусловливает неутихающий интерес к теоретическому изучению эффектов локализации энергии световых пучков, распространяющихся вдоль границ раздела в слоистых средах, играющих роль оптических волноводов $[13,14]$. Достаточно хорошо изученными являются эффекты локализации электромагнитного поля вблизи границ раздела сред с одинаковыми по физической природе механизмами формирования нелинейного отклика. Наиболее часто встречающиеся случаи относятся к описанию нелинейных поверхностных волн, распространяющихся вдоль границ раздела сред с керровской нелинейностью [6,15-19], либо границ между линейной и нелинейной средами [20-23].

В связи с этим возникает необходимость детального анализа вопросов существования нелинейных поверхностных волн, возбуждение которых может происходить вблизи границ раздела кристаллов с принципиально различными формами нелинейности, в частности в слоистых структурах, состоящих из чередующихся кристаллов с фоторефрактивным эффектом и эффектом Керра. Поэтому в настоящей работе предлагается теоретическое описание новых типов нелинейных поверхностных волн, распространяющихся вдоль границ трехслойной структуры, в которой внутренняя прослойка (пластина) представляет собой кристалл с керровской фокусирую- 
щей нелинейностью, а ее внешние обкладки - фоторефрактивные кристаллы. Различия форм нелинейности слоев, обусловленные различными механизмами индуцирования поля вследствие перераспределения плотности зарядов в них, приводит к возможности появления принципиально новых особенностей формирования профиля нелинейных поверхностных волн, амплитуда поля в которых будет убывать при удалении от границ раздела во внешние слои осциллирующим образом [24-27]. В трехслойных структурах, в которых внешние слои или линейные, или нелинейные с эффектом Керра, такого явления убывания амплитуды поверхностной волны с осцилляциями не наблюдается [4,28-37]. Убывающие с осцилляциями нелинейные поверхностные волны могут распространяться только в таких трехслойных структурах, в которых внешние слои состоят из сред с фоторефрактивным эффектом.

В настоящей работе будет рассматриваться только симметричная трехслойная структура, внешние слои которой состоят из сред с одинаковыми оптическими характеристиками. В силу симметрии в такой системе могут возникать нелинейные поверхностные волны с распределением профиля поля, характеризующимся определенной симметрией относительно границ раздела слоев, в частности синфазные и противофазные, описываемые четными и нечетными решениями соответственно.

\section{Уравнения модели}

Будем изучать распространение нелинейных поверхностных волн с необыкновенной поляризацией (ТМ-волны или Р-поляризованные), для которых $E_{y}=0$, $H_{x}=H_{z}=0$, в трехслойной структуре, представляющей собой пластину толщины $2 a$ из одноосного кристалла, обладающего фокусирующей нелинейностью керровского вида, зажатую между одноосными фоторефрактивными кристаллами с диффузионным механизмом формирования нелинейности в отсутствие приложенного внешнего поля. Границы раздела кристаллических слоев будем считать настолько тонкими, что можно будет пренебречь оптическими эффектами внутри них.

Систему координат выберем так, чтобы средина пластины нелинейного керровского кристалла проходила через начало координат, а границы раздела слоев лежали в плоскостях $x= \pm a$ перпендикулярно оси $x$. Тогда фоторефрактивные кристаллы занимают полупространства $|x|>a$, а пластина керровского кристалла расположена в области $|x|<a$. Полярные оси фоторефрактивных кристаллов будем считать ориентированными вдоль оси $x$ в противоположных направлениях.

Пусть световой пучок с интенсивностью, независящей от координаты $y$, распространяется вдоль границ раздела слоев, расположенных в плоскостях $x= \pm a$, параллельно оси $z$. Так как будет рассматриваться скользящее распространение светового пучка, то можно пренебречь анизотропией показателя преломления и использовать одноосное приближение.

Будем рассматривать только стационарное распределение поля поверхностной волны. Из системы уравнений Максвелла в рассматриваемом случае получается уравнение для отличной от нуля компоненты вектора напряженности магнитного поля [25-27]:

$$
\frac{\partial^{2} H_{y}}{\partial x^{2}}+\frac{\partial^{2} H_{y}}{\partial z^{2}}+k_{0}^{2} n^{2}(x) H_{y}=0,
$$

где $k_{0}=2 \pi / \lambda_{0}, \lambda_{0}$ - длина волны света в вакууме, $n(x)$ - пространственное распределение изменения показателя преломления света вдоль трехслойной структуры, т.е. в направлении, перпендикулярном границам раздела слоев. Будем аппроксимировать данную зависимость функцией

$$
n(x)= \begin{cases}n_{P 0}+\Delta n_{P}(x), & |x|>a \\ n_{K 0}+\Delta n_{K}(x), & |x|<a\end{cases}
$$

$n_{j 0}$ - невозмущенные показатели преломления, $\Delta n_{j}-$ нелинейные добавки к ним, которые считаются малыми $\left(\Delta n_{j} \ll n_{j 0}\right), j=P, K$. Здесь и далее значение индекса $j=P$ соответствует величинам, описывающим свойства фоторефрактивных кристаллов в области $|x|>a$, а значение индекса $j=K$ соответствует величинам, описывающим свойства керровского кристалла в пластине при $|x|<a$.

Будем считать, что нелинейная добавка к невозмущенному показателю преломления в фоторефрактивных кристаллах формируется в результате только диффузионного механизма нелинейности [11]. Тогда в приближении полного отсутствия темновой (фоновой) засветки нелинейную добавку к показателю преломления фоторефрактивных кристаллов можно представить в виде [11,25-27]

$$
\Delta n_{P}(x)=\frac{1}{2} n_{P 0}^{3} r_{e f f} \frac{k_{B} T}{e} \frac{I^{\prime}}{I},
$$

где штрихи здесь и далее означают производные по координате $x, r_{e f f}-$ эффективный электрооптический коэффициент, $k_{B}$ - константа Больцмана, $T-$ температура, $e-$ модуль заряда электрона, $I \propto\left|H_{y}\right|^{2}-$ интенсивность света в поверхностной волне.

Нелинейная добавка к показателю преломления керровского кристалла пропорциональна интенсивности света в поверхностной волне и тогда для нее будем использовать выражение в виде

$$
\Delta n_{K}(x)=\alpha\left|H_{y}\right|^{2},
$$

$\alpha-$ коэффициент керровской нелинейности, который постоянный и положительный для фокусирующей среды.

Пусть установившееся распределение распространяющейся вдоль оси $z$ волны представимо в виде

$$
H_{y}(x, z)=e^{i \beta k_{0} z} \begin{cases}H_{1}(x), & x<-a ; \\ H_{K}(x), & |x|<a \\ H_{2}(x), & x>a\end{cases}
$$


где $\beta$ - константа распространения. Тогда в рассматриваемом приближении с учетом отсутствия темновой засветки и малости нелинейных добавок к невозмущенным показателям преломления из (1)-(4) можно получить уравнения

$$
\begin{gathered}
H_{1,2}^{\prime \prime}+\mu H_{1,2}^{\prime}+\left(n_{P 0}^{2}-\beta^{2}\right) k_{0}^{2} H_{1,2}=0, \\
H_{K}^{\prime \prime}+\left(n_{K 0}^{2}-\beta^{2}\right) k_{0}^{2} H_{K}+g\left|H_{K}\right|^{2}=0,
\end{gathered}
$$

где обозначили $\mu=2 k_{0}^{2} n_{P 0}^{4} r_{e f f} k_{B} T / e-$ коэффициент затухания волны в фоторефрактивных кристаллах, названный по аналогии уравнения (6) с гармоническим осциллятором с затуханием [27], $g=2 \alpha k_{0}^{2} n_{K 0}-$ коэффициент нелинейности в керровском кристалле, который для рассматриваемой в настоящей работе фокусирующей нелинейности является положительной величиной. Для существования поверхностных волн толщина слоев фоторефрактивных кристаллов должна быть много больше обратного коэффициента затухания, фактически определяющего глубину проникновения поля. Кроме того, используемое приближение, а также уравнения (6) и (7) справедливы только областях фоторефрактивного кристалла, расположенных за пределами узкого приграничного слоя порядка длины экранировки Дебая, где не учитываются эффекты, связанные с накоплением экранирующего заряда.

Из условий непрерывности тангенциальных составляющих полей на границах раздела слоев вытекают граничные условия:

$$
\begin{gathered}
H_{1}(-a)=H_{K}(-a), \quad H_{2}(a)=H_{K}(a), \\
\frac{1}{n_{P 0}^{2}} H_{1}^{\prime}(-a)=\frac{1}{n_{K 0}^{2}} H_{K}^{\prime}(-a), \quad \frac{1}{n_{P 0}^{2}} H_{2}^{\prime}(a)=\frac{1}{n_{K 0}^{2}} H_{K}^{\prime}(a) .
\end{gathered}
$$

Таким образом, математическая формулировка модели для описания нелинейных поверхностных волн в трехслойной структуре из пластины с керровской нелинейностью и внешних обкладок из фоторефрактивных кристаллов сводится к уравнениям (6) и (7) с граничными условиями (8) и (9). Нелинейные поверхностные волны в рассматриваемой модели представляют собой решения контактной краевой задачи (6)-(9), удовлетворяющие условиям ограниченности.

Поскольку в данной модели предполагается, что все оптические характеристики фоторефрактивных кристаллов слева и справа от пластины одинаковы, то в такой симметричной трехслойной структуре могут возникать поверхностные состояния, обладающие симметрией распределения поля, которые описываются четными $\left(H_{1}(x)=H_{2}(-x)\right)$ и нечетными $\left(H_{1}(x)-H_{2}(-x)\right)$ решениями. В настоящей работе будут рассматриваться поверхностные состояния только такого вида, поэтому в дальнейшем будет использоваться обозначение для напряженности магнитного поля в фоторефрактивном кристалле $H_{P}(x)=H_{1}(x)$ при $x<-a$, а в области $x>a$ поле $H_{P}(x)$ будет продолжаться четным или нечетным образом.
Уравнение (6) имеет два типа исчезающих на бесконечности решений в зависимости от соотношения между значениями константы распространения, коэффициента затухания и невозмущенного показателя преломления в фоторефрактивном кристалле. Амплитуда волны первого типа убывает без осцилляций при удалении от границ раздела слоев вглубь фоторефрактивных кристаллов, а второго - осциллирующим образом [25].

Решения нелинейного уравнения (7) определяются знаком коэффициента нелинейности $g$ и знаком разности $n_{K 0}^{2}-\beta^{2}$. В зависимости от их комбинаций возникает несколько типов нелинейных поверхностных волн. В рассматриваемой модели симметричной трехслойной структуры для фокусирующего внутреннего слоя при $g>0$ будут использоваться периодические решения, которые описываются эллиптическими функциями Якоби cn и dn, что приводит к существованию двух наборов нелинейных поверхностных волн, различающихся формой распределения поля в керровском кристалле. В каждом из таких наборов, помимо вида симметрии поверхностного состояния, встречаются различные формы затухания поля при удалении от границ раздела во внешние слои фоторефрактивных кристаллов.

\section{Поверхностные волны с убывающей без осцилляций амплитудой}

\section{Первый тип волн с симметричным распределением поля}

Профиль распределения поля в фоторефрактивном кристалле в области $x<-a$ описывается решением уравнения (6), которое при $\beta>\sqrt{n_{P 0}^{2}-\mu^{2} / 4 k_{0}^{2}}$ представимо в виде

$$
H_{P}(x)=A e^{(\mu / 2+v)(x+a)}+B e^{(\mu / 2-v)(x+a)},
$$

где

$$
v^{2}=\mu^{2} / 4-k_{0}^{2}\left(n_{P 0}^{2}-\beta^{2}\right) .
$$

Для волны с симметричным относительно центра трехслойной структуры распределением профиля поля (10) продолжается четным образом в область $x>a$ :

$$
H_{P}(x)=A e^{-(\mu / 2+v)(x-a)}+B e^{-(\mu / 2-v)(x-a)} .
$$

Внутри пластины из керровского кристалла профиль поля является периодически распределенным в пространстве и описывается для волн первого типа периодическим четным решением уравнения (7) при $g>0$ и $\beta>n_{K 0}$ :

$$
H_{K}(x)=k q_{c} \sqrt{\frac{2}{g}} \operatorname{cn}\left(q_{c} x, k\right),
$$

где

$$
q_{c}^{2}=k_{0}^{2}\left(\beta^{2}-n_{K 0}^{2}\right) /\left(2 k^{2}-1\right),
$$

$k$ - модуль эллиптической функции Якоби, $1 / 2<k^{2}<1$. 
Пространственный период осцилляций распределения поля в пластине из керровского кристалла, согласно (12), $\Lambda_{K c}=4 K(k) / q_{c}$, где $K(k)$ - полный эллиптический интеграл первого рода.

Подстановка решений (10) и (12) в граничные условия (8) и (9) приводит к выражениям для параметров поля в фоторефрактивном кристалле:

$$
\begin{aligned}
& A=\frac{k q_{c} \operatorname{cn}\left(q_{c} a, k\right)}{v \sqrt{2 g}}\left(\gamma_{c e}-\frac{\mu}{2}+v\right), \\
& B=\frac{k q_{c} \operatorname{cn}\left(q_{c} a, k\right)}{v \sqrt{2 g}}\left(\frac{\mu}{2}+v-\gamma_{c e}\right),
\end{aligned}
$$

где

$$
\gamma_{c e}=q_{c} \frac{n_{P 0}^{2}}{n_{K 0}^{2}} \frac{\operatorname{sn}\left(q_{c} a, k\right) \operatorname{dn}\left(q_{c} a, k\right)}{\operatorname{cn}\left(q_{c} a, k\right)} .
$$

Величина $k$ здесь играет роль свободного параметра, которая может быть определена при заданном значении потока энергии, переносимого поверхностной волной.

Таким образом, нелинейная поверхностная волна с неосциллирующим профилем описывается симметричным распределением полей (10) и (12) с параметрами, определяемыми выражениями (11), (13)-(16), и существует при значениях константы распространения $\beta>\max \left\{n_{K 0}, \sqrt{n_{P 0}^{2}-\mu^{2} / 4 k_{0}^{2}}\right\}$.

Как отмечалось в [26], поверхностная волна с неосциллирующим профилем амплитуды может наблюдаться только при очень малых углах скольжения пучка, возбуждающего эту волну. При очень малых углах падения возбуждающего пучка на входной торец кристалла фоторефрактивная решетка формируется под действием света только в тех областях фоторефрактивного кристалла, в которых интенсивность световой волны мала по сравнению с темновой интенсивностью.

Нелинейная поверхностная волна с неосциллирующим профилем (10) может убывать в глубину фоторефрактивного кристалла немонотонно или монотонно.

Поверхностная волна с немонотонным профилем может иметь максимумы, симметрично расположенные на расстояниях

$$
x_{m}=\frac{1}{2} \ln \left(\frac{(v-\mu)\left(v+\mu / 2-\gamma_{c e}\right)}{(v+\mu)\left(v-\mu / 2+\gamma_{c e}\right)}\right)
$$

от границ раздела слоев. Значение поля на таких расстояниях может существенно превосходить амплитуду поля на границах раздела слоев $H_{a}=H_{P}( \pm a)=A+B$.

Монотонное убывание амплитуды волны в глубину фоторефрактивных кристаллов происходит в двух случаях: 1) $A=0$ и тогда $H_{a}=B$ или 2) $B=0$ и тогда $H_{a}=A$.

Тогда из (14) и (15) получаются дисперсионные уравнения

$$
\gamma_{c e}=\mu / 2 \pm v
$$

где выбирается знак ,-““ для $A=0$ и „,+“ для $B=0$.

В этих случаях симметричное распределение поля (10) поверхностной волны с монотонной убывающей амплитудой в фоторефрактивных кристаллах, принимает вид

$$
H_{P}(x)=H_{a} e^{ \pm \gamma_{c e}(x \pm a)},
$$

где амплитуда поля на границах раздела слоев

$$
H_{a}=\sqrt{2 / g} k q_{c} \operatorname{cn}\left(q_{c} a, k\right) .
$$

Величина $\gamma_{c e}$, определяемая выражением (16), имеет смысл коэффициента убывания нелинейной поверхностной волны, обратно пропорционального глубине проникновения поля в фоторефрактивные кристаллы. Так как коэффициент убывания должен быть положительным, то возникают ограничения для значений константы распространения для монотонно затухающей волны.

Дисперсионное уравнение (18) определяет такую связь константы распространения с оптическими характеристиками трехслойной структуры, при которой будет наблюдаться монотонное убывание профиля поля нелинейной поверхностной волны при удалении от границ раздела в глубину фоторефрактивных кристаллов.

В приближении $q_{c} a \ll 1$, которое будем условно называть далее длинноволновым, из дисперсионного уравнения (18) получается зависимость константы распространения от оптических характеристик трехслойной структуры (закон дисперсии) в явном виде

$$
\beta^{2}=\frac{n_{P 0}^{2} n_{K 0}^{2}\left(2 k^{2}-1+a \mu\right)}{n_{K 0}^{2}\left(2 k^{2}-1\right)+n_{P 0}^{2} a \mu} .
$$

Длинноволновые симметричные колебательные состояния поля в рассматриваемой трехслойной структуре существуют без дополнительных ограничений для ее оптических характеристик.

\section{Второй тип волн с симметричным распределением поля}

Для волн второго типа с симметричным распределением внутри пластины из керровского кристалла профиль поля может описываться периодически распределенным в пространстве четным решением уравнения (7) второго типа при $g>0$ и $\beta>n_{K 0}$ :

$$
H_{K}(x)=q_{d} \sqrt{\frac{2}{g}} \mathrm{dn}\left(q_{d} x, k\right),
$$

где

$$
q_{d}^{2}=k_{0}^{2}\left(\beta^{2}-n_{K 0}^{2}\right) /\left(2-k^{2}\right),
$$

$0<k^{2}<1$.

Профиль распределения поля в фоторефрактивном кристалле в области $x<-a$ при $\beta>\sqrt{n_{P 0}^{2}-\mu^{2} / 4 k_{0}^{2}}$ описывается выражением (10), продолжаемым четным образом для симметричного состояния в область $x>a$.

Пространственный период осцилляций поля в пластине из керровского кристалла, согласно (22), $\Lambda_{K d}=4 K(k) / q_{d}$. 
Подстановка решений (10) и (22) в граничные условия (8) и (9) приводит к следующим выражениям для параметров поля в фоторефрактивном кристалле:

$$
\begin{aligned}
& A=\frac{q_{d} \operatorname{dn}\left(q_{d} a, k\right)}{v \sqrt{2 g}}\left(\gamma_{d e}-\frac{\mu}{2}+v\right), \\
& B=\frac{q_{d} \operatorname{dn}\left(q_{d} a, k\right)}{v \sqrt{2 g}}\left(\frac{\mu}{2}+v-\gamma_{d e}\right),
\end{aligned}
$$

где

$$
\gamma_{d e}=q_{d} k^{2} \frac{n_{P 0}^{2}}{n_{K 0}^{2}} \frac{\operatorname{sn}\left(q_{d} a, k\right) \operatorname{cn}\left(q_{d} a, k\right)}{\operatorname{dn}\left(q_{d} a, k\right)},
$$

Монотонное убывание амплитуды волны в глубину фоторефрактивных кристаллов происходит при зависимости константы распространения от оптических характеристик трехслойной структуры, определяемой из дисперсионных уравнений

$$
\gamma_{d e}=\mu / 2 \pm v
$$

где выбирается знак „,-“ для $A=0$ и „,+“ для $B=0$.

Симметричное распределение поля поверхностной волны, с монотонной убывающей амплитудой в фоторефрактивных кристаллах, имеет вид

$$
H_{P}(x)=H_{a} e^{ \pm \gamma_{d e}(x \pm a)},
$$

где амплитуда поля на границах раздела слоев

$$
H_{a}=\sqrt{2 / g} q_{d} \operatorname{dn}\left(q_{d} a, k\right) .
$$

В отличие от амплитуды поля на границах раздела слоев волн первого типа, определяемой (20), амплитуда волн второго типа (29) не обращается в ноль.

В длинноволновом приближении $q_{d} a \ll 1$ из дисперсионного уравнения (28) получается зависимость константы распространения от оптических характеристик трехслойной структуры в явном виде:

$$
\beta^{2}=\frac{n_{P 0}^{2} n_{K 0}^{2}\left(2-k^{2}+a \mu k^{2}\right)}{n_{K 0}^{2}\left(2-k^{2}\right)+n_{P 0}^{2} a \mu k^{2}} .
$$

Длинноволновые симметричные колебательные состояния поля в рассматриваемой трехслойной структуре существуют при выполнении условий:

1) если невозмущенный показатель преломления в фоторефрактивных кристаллах больше невозмущенного показателя преломления в керровском кристалле, то эллиптический модуль должен удовлетворять условию $k^{2}<2 /(1-a \mu)$ и ширина пластины керровского кристалла должна быть меньше характерной глубины проникновения волны в фоторефрактивный кристалл: $2 a<l=2 / \mu$

2) если выполняется противоположное условие, т.е. $n_{P 0}<n_{K 0}$, то эллиптический модуль должен удовлетворять условию $k^{2}<2 /\left(1-a \mu k^{2} n_{P 0}^{2} / n_{K 0}^{2}\right)$, а ширина пластины керровского кристалла должна быть связана с коэффициентом затухания в фоторефрактивном кристалле и невозмущенными показателями преломления слоев условием $a<n_{K 0}^{2} / \mu n_{P 0}^{2}$.

Данные условия следует учитывать при проектировании устройств, использующих трехслойные структуры рассматриваемого вида. Если невозмущенный показатель преломления во внешних слоях из фоторефрактивных кристаллов меньше невозмущенного показателя преломления внутренней пластины из керровского кристалла, то ее ширина может быть выбрана больше, чем при обратном условии, для того, чтобы добиться монотонного убывания поверхностной волны.

\section{Волны с антисимметричным распределением поля}

Для волн с антисимметричным распределением внутри пластины из керровского кристалла профиль поля описывается периодически распределенным в пространстве периодическим нечетным решением уравнения (7) первого типа при $g>0$ и $\beta>n_{K 0}$ :

$$
H_{K}(x)=-k_{1} k q_{c} \sqrt{\frac{2}{g}} \frac{\operatorname{sn}\left(q_{c} x, k\right)}{\operatorname{dn}\left(q_{c} x, k\right)},
$$

где $k_{1}^{2}=1-k^{2}-$ дополнительный модуль.

Пространственный период осцилляций поля в пластине из керровского кристалла, согласно (31), такой же, как для поля, описываемого четным решением (12).

Профиль распределения поля в фоторефрактивном кристалле в области $x<-a$ при $\beta>\sqrt{n_{P 0}^{2}-\mu^{2} / 4 k_{0}^{2}}$ описывается выражением (10), продолжаемым нечетным образом для антисимметричного состояния в область $x>a$ :

$$
H_{P}(x)=-A e^{-(\mu / 2+v)(x-a)}-B e^{-(\mu / 2-v)(x-a)} .
$$

Подстановка решений (10) и (31) в граничные условия (8) и (9) приводит к следующим выражениям для параметров поля в фоторефрактивном кристалле:

$$
\begin{aligned}
& A=\frac{k k_{1} q_{c} \operatorname{sn}\left(q_{c} a, k\right)}{v \sqrt{2 g} \operatorname{dn}\left(q_{c} a, k\right)}\left(\gamma_{c o}-\frac{\mu}{2}+v\right), \\
& B=\frac{k k_{1} q_{c} \operatorname{sn}\left(q_{c} a, k\right)}{v \sqrt{2 g} \operatorname{dn}\left(q_{c} a, k\right)}\left(\frac{\mu}{2}+v-\gamma_{c o}\right),
\end{aligned}
$$

где

$$
\gamma_{c o}=q_{c} \frac{n_{P 0}^{2}}{n_{K 0}^{2}} \frac{\operatorname{cn}\left(q_{c} a, k\right)}{\operatorname{sn}\left(q_{c} a, k\right) \operatorname{dn}\left(q_{c} a, k\right)} .
$$

Монотонное убывание волны в глубину фоторефрактивных кристаллов происходит при зависимости константы распространения от оптических характеристик трехслойной структуры, определяемой из дисперсионных уравнений:

$$
\gamma_{c o}=\mu / 2 \pm v,
$$

где выбирается знак „,-“ для $A=0$ и „,+“ для $B=0$. 
Антисимметричное распределение поля поверхностной волны, монотонной убывающей в фоторефрактивных кристаллах, имеет вид

$$
H_{P}(x)= \pm H_{a} e^{ \pm \gamma_{c o}(x \pm a)},
$$

где амплитуда поля на границах раздела слоев

$$
H_{a}=-\sqrt{2 / g} k k_{1} \operatorname{sn}\left(q_{c} a, k\right) / \operatorname{dn}\left(q_{c} a, k\right) .
$$

В длинноволновом приближении $q_{c a} \ll 1$ из дисперсионного уравнения (35) получается зависимость константы распространения от оптических характеристик трехслойной структуры в явном виде:

$$
\beta^{2}=n_{P 0}^{2}\left\{1-\frac{1}{a k_{0}^{2} n_{K 0}^{2}}\left(a \mu-\frac{n_{P 0}^{2}}{n_{K 0}^{2}}\right)\right\} .
$$

Длинноволновые антисимметричные колебательные состояния поля в рассматриваемой трехслойной структуре существуют, если ширина пластины керровского кристалла связана с коэффициентом затухания в фоторефрактивном кристалле и невозмущенными показателями преломления слоев условием: $a>n_{P 0}^{2} / \mu n_{K 0}^{2}$.

Следует отметить, что в длинноволновом приближении $q_{c a} \ll 1$ из (35) получается оценка коэффициента убывания $\gamma_{c o}=n_{P 0}^{2} / a n_{K 0}^{2}$. Отсюда вытекает, что глубиной проникновения поверхностной волны длинноволнового диапазона во внешние слои можно напрямую управлять, подбирая ширину внутренней пластины и соотношение между невозмущенными показателями преломления слоев трехслойной структуры, поскольку она определяется выражением $l_{c 0}=1 / \gamma_{c 0}=a n_{K 0}^{2} / n_{P 0}^{2}$. Следовательно, вблизи внутреннего слоя, состоящего из более узкой внутренней пластины трехслойной структуры, поверхностная волна будет распространяться, локализуя бо́льшую долю энергии в более узких приповерхностных слоях. Такого же эффекта сужения пространственной локализации энергии поверхностной волны можно добиться, если подобрать внутренний (керровский) оптический слой с показателем преломления меньшим, чем во внешних (фоторефрактивных) слоях трехслойной структуры.

\section{Поверхностные волны с убывающей с осцилляциями амплитудой}

\section{Осциллирующие поверхностные волны первого типа с симметричным распределением поля}

Симметричное распределение профиля поля, убывающего с осцилляциями в фоторефрактивном кристалле в области $x<-a$, описывается решением уравнения (6), которое при $\beta<\sqrt{n_{P 0}^{2}-\mu^{2} / 4 k_{0}^{2}}$ представимо в виде

$$
H_{P}(x)=H_{a} e^{\mu(x+a) / 2} \cos (p(x+a)-\varphi) / \cos \varphi,
$$

где амплитуда поля на границе раздела слоев определяется выражением (20), и волновое число

$$
p^{2}=-v^{2}=k_{0}^{2}\left(n_{P 0}^{2}-\beta^{2}\right)-\mu^{2} / 4 .
$$

Для волны с симметричным относительно центра трехслойной структуры распределением профиля поля (39) продолжается четным образом в область $x>a$ :

$$
H_{P}(x)=H_{a} e^{-\mu(x-a) / 2} \cos (p(x-a)+\varphi) / \cos \varphi .
$$

Поверхностная волна с осциллирующим профилем амплитуды наблюдалась при наклонном падении сфокусированного пучка света на полированный торец кристалла [27].

Пространственный период осцилляций убывания поля в фоторефрактивных кристаллах $\Lambda_{P}=2 \pi / p$. При этом волна проникает в глубину фоторефрактивных кристаллов на расстояние $l=2 / \mu=e / n_{P 0}^{4} r_{e f f} k_{B} T$. К примеру, для характерных значений параметров фоторефрактивных кристаллов типа SBN $n_{p}=2.3, r_{e f f}=750 \mathrm{pm} / \mathrm{V}$ при температуре $T=300^{\circ} \mathrm{C}$ получается оценка глубины проникновения поля на расстояние порядка $l=2.16 \mu \mathrm{m}$ от границ раздела, а при температуре $T=600^{\circ} \mathrm{C}-$ $l=1.42 \mu \mathrm{m}$. Оценка глубины локализации поля в приграничных слоях согласуется с экспериментальными значениями $[38,39]$. Оценка глубины проникновения поля превосходит на порядок длину волны лазера, возбуждающего поверхностную волну (порядка $0.4 \mu \mathrm{m}$ [25]). На таких сравнительно небольших расстояниях можно считать, что используемое приближение отсутствия темновой засветки остается справедливым в типичных экспериментах при интенсивностях возбуждающего лазерного излучения порядка $I \approx 140 \mathrm{~W} / \mathrm{cm}^{2}$ [40], так как типичное для SBN кристаллов значение темновой интенсивности $I_{d} \approx 0.03 \mathrm{~W} / \mathrm{cm}^{2}$ [27] пренебрежительно мало, что и оправдывает применение приближения диффузионного механизма формирования нелинейности с малым возмущением показателя преломления, согласно (3), на расстояниях порядка нескольких длин волн от границ.

Для первого типа волн с симметричным распределением внутри пластины из керровского кристалла профиль поля при $g>0$ и $\beta>n_{K 0}$ описывается попрежнему выражением (12).

Таким образом, поверхностная волна с убывающей с осцилляциями амплитудой описывается симметричным распределением полей (12) и (39) и существует при значениях константы распространения, лежащих в диапазоне $N_{K 0}<\beta<\sqrt{n_{P 0}^{2}-\mu^{2} / 4 k_{-}^{2}}$.

Подстановка решений (12) и (39) в граничные условия (8) и (9) приводит к дисперсионному соотношению

$$
\frac{\mu}{2}+p \operatorname{tg} \varphi=\gamma_{c e},
$$

где коэффициент убывания $\gamma_{c e}$ определяется выражением (16).

Значение фазы $\varphi=0$ соответствует случаю, когда коэффициенты связаны соотношением $\gamma_{c e}=\mu / 2$. 
Пространственный период осцилляций убывания поля во внешних слоях из фоторефрактивных кристаллов связан с коэффициентами затухания .

$$
\Lambda_{p}=2 \pi \operatorname{tg} \varphi /\left(\gamma_{c e}-\mu / 2\right)
$$

В поверхностной волне, амплитуда в которой убывает с осцилляциями, от константы распространения зависит только период осцилляций и не зависит глубина ее проникновения во внешние слои трехслойной структуры. Глубина проникновения поверхностной волны во внешние слои может регулироваться оптическими характеристиками только фоторефрактивных кристаллов. В частности, чем больше невозмущенный показатель преломления или электрооптический коэффициент фоторефрактивного кристалла, тем меньше будет данная глубина проникновения. В отличие от глубины проникновения период пространственных осцилляций можно регулировать не только оптическими характеристиками фоторефрактивных кристаллов, но и керровского, в частности, толщиной внутреннего слоя и невозмущенным показателем преломления керровского кристалла.

В длинноволновом приближении $q_{c} a \ll 1$ из дисперсионного уравнения (41) получается зависимость константы распространения от оптических характеристик трехслойной структуры в явном виде:

$$
\beta^{2}=\frac{k_{0}^{2} n_{P 0}^{2}\left\{a \mu /\left(2 k^{2}-1\right)-\operatorname{tg}^{2} \varphi\right\}+\mu^{2}\left(1+\operatorname{tg}^{2} \varphi\right) / 4}{k_{0}^{2}\left\{n_{P 0}^{2} a \mu / n_{K 0}^{2}\left(2 k^{2}-1\right)-\operatorname{tg}^{2} \varphi\right\}} .
$$

Для существования такой волны должно выполняться условие

$$
\operatorname{tg}^{2} \varphi<\min \left\{\frac{k_{0}^{2} n_{P 0}^{2} a \mu /\left(2 k^{2}-1\right)+\mu / 4}{k_{0}^{2} n_{P 0}^{2}-\mu^{2} / 4}, \frac{n_{P 0}^{2} a \mu}{n_{K 0}^{2}\left(2 k^{2}-1\right)}\right\} .
$$

В частном случае при $\varphi=0$ (42) можно привести к простому виду

$$
\beta^{2}=n_{K 0}^{2}\left\{1+\frac{\left(2 k^{2}-1\right) \mu}{2 a k_{0}^{2} n_{P 0}^{2}}\right\} .
$$

При такой зависимости константы распространения поверхностная волна с убывающими с осцилляциями симметричным профилем амплитуды поля в длинноволновом диапазоне может существовать без дополнительных ограничений для оптических характеристик трехслойной структуры рассматриваемого вида.

\section{Осциллирующие поверхностные волны второго типа с симметричным распределением поля}

Профиль распределения поля в фоторефрактивном кристалле в области $x<-a$ при $\beta<s \sqrt{n_{P 0}^{2}-\mu^{2} / 4 k_{0}^{2}}$ описывается выражением (39) с амплитудой (29), продолжаемым четным образом для симметричного состояния в область $x>a$.
Для волн второго типа с симметричным распределением внутри пластины из керровского кристалла при $g>0$ и $\beta>n_{K 0}$ профиль поля описывается выражением (22).

Подстановка решений (22) и (39) в граничные условия (8) и (9) приводит к дисперсионному соотношению

$$
\frac{\mu}{2}+p \operatorname{tg} \varphi=\gamma_{d e},
$$

где коэффициент затухания $\gamma_{d e}$ определяется выражением (26).

Значение фазы $\varphi=0$ соответствует случаю, когда коэффициенты затухания связаны $\gamma_{d e}=\mu / 2$.

Пространственный период осцилляций убывания поля во внешних слоях из фоторефрактивных кристаллов связан с коэффициентами убывания волны второго типа и определяется выражением $\Lambda_{P}=2 \pi \operatorname{tg} \varphi /\left(\gamma_{d e}-\mu / 2\right)$.

В длинноволновом приближении $q_{d} a \ll 1$ из дисперсионного уравнения (44) получается зависимость константы распространения от оптических характеристик трехслойной структуры в явном виде:

$$
\beta^{2}=\frac{n_{P 0}^{2}\left\{\operatorname{tg}^{2} \varphi-a \mu k^{2} /\left(2-k^{2}\right)\right\}-\mu^{2}\left(1+\operatorname{tg}^{2} \varphi\right) / 4 k_{0}^{2}}{\operatorname{tg}^{2} \varphi-a \mu k^{2} n_{P 0}^{2} / n_{K 0}^{2}\left(2-k^{2}\right)} .
$$

Для существования такой волны должно выполняться условие

$$
\operatorname{tg}^{2} \varphi>\max \left\{\frac{a \mu k^{2} k_{0}^{2} n_{P 0}^{2} /\left(2-k^{2}\right)+\mu^{2} / 4}{k_{0}^{2} n_{p 0}^{2}-\mu^{2} / 4}, \frac{a \mu k^{2} n_{P 0}^{2}}{n_{K 0}^{2}\left(2-k^{2}\right)}\right\} .
$$

В частном случае при $\varphi=0$ (45) можно привести к простому виду

$$
\beta^{2}=n_{K 0}^{2}\left\{1+\frac{\left(2-k^{2}\right) \mu}{2 a k_{0}^{2} k^{2} n_{P 0}^{2}}\right\} .
$$

При такой зависимости константы распространения волна второго типа, как и первого, может существовать без дополнительных ограничений для оптических характеристик трехслойной структуры.

\section{Осциллирующие поверхностные волны с антисимметричным распределением поля}

При $g>0$ и $n_{K 0}<\beta<\sqrt{n_{P 0}^{2}-\mu^{2} / 4 k_{0}^{2}}$ существует поверхностная волна с антисимметричным распределением, в которой профиль поля внутри пластины из керровского кристалла описывается выражением (31), а профиль поля в фоторефрактивном кристалле в области $x<-a$ описывается выражением (39) с амплитудой (37), продолжаемым нечетным образом в область $x>a$ :

$$
H_{P}(x)=-H_{a} e^{-\mu(x-a) / 2} \cos (p(x-a)+\varphi) / \cos \varphi .
$$


Подстановка решений (31) и (39) в граничные условия (8) и (9) приводит к дисперсионному соотношению

$$
\frac{\mu}{2}+p \operatorname{tg} \varphi=\gamma_{c o}
$$

где коэффициент убывания $\gamma_{c o}$ определяется выражением (34).

Из (47) следует, что пространственный период осцилляций убывания поля во внешних слоях из фоторефрактивных кристаллов связан с коэффициентами убывания волны с антисимметричным профилем: $\Lambda_{P}=2 \pi \operatorname{tg} \varphi /\left(\gamma_{c o}-\mu / 2\right)$.

В длинноволновом приближении $q_{c} a \ll 1$ из дисперсионного уравнения (47) получается зависимость константы распространения от оптических характеристик трехслойной структуры в явном виде:

$$
\beta^{2}=n_{P 0}^{2}-\frac{\mu^{2}}{4 k_{0}^{2}}-\frac{\operatorname{ctg}^{2} \varphi}{k_{0}^{2}}\left(\frac{\mu}{2}-\frac{n_{P 0}^{2}}{a n_{K 0}^{2}}\right)^{2} .
$$

При такой зависимости константы распространения убывающая с осцилляциями антисимметричная поверхностная волна в длинноволновом диапазоне может существовать без дополнительных ограничений для оптических характеристик трехслойной структуры рассматриваемого вида.

Пространственный период осцилляций убывания амплитуды поля в фоторефрактивных кристаллах для случая длинноволнового режима распространения поверхностной волны с антисимметричным профилем определяется выражением $\Lambda_{P}=2 \pi \operatorname{tg} \varphi /\left(n_{P 0}^{2}-a \mu n_{K 0}^{2} / 2\right)$. Отсюда следует, что при сравнительно малых толщине внутренней пластины и отношении невозмущенных показателей преломления внутреннего и внешних слоев, когда выполняется условие их малости по сравнению с глубиной проникновения поверхностной волны во внешние слои, $a n_{K 0}^{2} / n_{P 0}^{2} \ll l$, пространственный период осцилляций можно оценить по формуле $\Lambda_{P}=2 \pi a n_{K 0}^{2} \operatorname{tg} \varphi / n_{P 0}^{2}$. Следовательно, выбирая более узким внутренний слой из керровского кристалла, можно уменьшить период осцилляций затухания поля в длинноволновом режиме распространения такой поверхностной волны вдоль трехслойной структуры рассматриваемого вида.

\section{Заключение}

В настоящей работе установлено, что вдоль трехслойной структуры, состоящей из фоторефрактивных кристаллов с одинаковыми оптическими свойствами, разделенных пластиной из кристалла, характеризующегося керровской фокусирующей нелинейностью, могут распространяться нелинейные поверхностные волны, различающиеся характером убывания амплитуды. Амплитуда волны одного вида убывает при удалении от границ раздела без осцилляций в глубину внешних слоев из фоторефрактивных кристаллов, а другого - затухает с осцилляциями. Амплитуда волны первого вида может убывать монотонно при определенных условиях связи константы распространения, коэффициентов преломления и других физических характеристик слоев.

В силу симметричного распределения физических характеристик трехслойной структуры вдоль нее распространяются нелинейные поверхностные волны с профилями двух форм симметрии: симметричные, описываемые четными функциями, и антисимметричные, описываемые нечетными функциями. Показано, что волны с амплитудами, убывающими как с осцилляциями, так и без них, с симметричным распределением профиля поля относительно центра трехслойной структуры существуют двух типов, а с антисимметричным - одного.

Для всех рассмотренных в работе типов нелинейных поверхностных волн получены дисперсионные уравнения. В явном аналитическом виде найдены зависимости константы распространения от характеристик слоистой структуры для длинноволнового режима распространения поверхностных волн, а также указаны условия их существования.

Показано, что в поверхностных волнах с амплитудой, затухающей без осцилляций, посредством подбора оптических параметров всех слоев и ширины внутреннего слоя трехслойной структуры можно регулировать глубину их проникновения во внешние фоторефрактивные слои и пространственный период осцилляций затухания поля в пластине из керровского кристалла.

В отличие от таких волн в поверхностных волнах, амплитуда в которых затухает с осцилляциями, глубина проникновения может регулироваться оптическими характеристиками только фоторефрактивных кристаллов внешних слоев. В частности, увеличение показателя преломления или электрооптического коэффициента фоторефрактивного кристалла влечет за собой уменьшение глубины проникновения поля поверхностной волны, приводящее к сужению пространственной локализации ее энергии вдоль слоев.

Для поверхностных волн с монотонно убывающей амплитудой такого же эффекта можно добиться, выбирая показатель преломления внутреннего (керровского) слоя меньше, чем во внешних (фоторефрактивных) слоях. Кроме того, к такому же эффекту приводит уменьшение толщины внутреннего слоя трехслойной структуры.

Следует отметить, что в трехслойной симметричной структуре помимо симметричных и антисимметричных поверхностных состояний поля могут существовать и состояния, не обладающие симметрией. В [29] отмечалось, что в симметричной трехслойной структуре, все слои которой характеризуются одинаковой фокусирующей керровской нелинейностью, могут существовать три вида поверхностных волн: с синфазными одинаковыми потоками в двух плоскостях границ раздела слоев, с противофазными одинаковыми потоками, а также с синфазными потоками разной интенсивности (амплитуды).

В рассматриваемой трехслойной структуре волны с несимметричным профилем могут быть описаны перио- 
дическими решениями нелинейного уравнения, к примеpy, вида $\operatorname{dn}\left(q_{d}\left(x-x_{0}\right), k\right)$ или другими эллиптическими функциями, где $x_{0} \neq 0$. Однако вопросы существования таких поверхностных волн требуют отдельного детального анализа, выходящего за рамки данной работы. Также следует отметить, что вопрос исследования устойчивости нелинейных волн требует отдельного рассмотрения и выходит за рамки данной работы.

Возможность существования волн с осциллирующим убыванием амплитуды поля принципиально отличает гетероструктуры на основе оптических сред с фоторефрактивным эффектом от слоистых структур, состоящих из других чередующихся оптических сред, нелинейных с эффектом Керра или линейных. Такие эффекты следует учитывать при разработке различных оптических устройств [41-47], основанных на структурах из чередующихся слоев кристаллов с различными механизмами формирования нелинейности и использующих их волноводные свойства.

\section{Конфликт интересов}

Авторы заявляют, что у них нет конфликта интересов.

\section{Список литературы}

[1] Qian Zh., Jin F., Lu T., Kishimoto K. // Acta Mechanica, 2009. V. 2007. P. 183. doi 10.1007/s00707-008-0123-6

[2] Panyaev I.S., Dadoenkova N.N., Dadoenkova Yu.S., Rozhleys I.A., Krawczyk M., Lyubchanckii I.L., Sannikov D.G. // J. Phys. D: Appl. Phys. 2016. V. 49 P. 435103. doi 10.1088/0022-3727/49/43/435103

[3] Trofimov V.A., Zakharova I.G., Shestakov P.Y. // Progress in Electromagnetics Research Symposium - Spring (PIERS). St. Petersburg, 2017. P. 3378. doi 10.1109/PIERS.2017.8262342

[4] Михалаке Д., Назмитдинов Р.Г., Федянин В.К. // Физика элементарных частиц и атомного ядра. 1989. Т. 20. С. 198.

[5] Sakaguchi H., Malomed B.A. // New J. Phys. 2016. V. 18. P. 025020. doi 10.1088/1367-2630/18/2/025020

[6] Дикштейн И.Е., Никитов С.А., Никитов И.Е. // ФТТ. 1998. T. 40. P. 1885. doi 10.1134/1.1130640; Dikshtein I.E., Nikitov S.A., Nikitov D.S. // Phys. Solid State. 1998. V. 40. P. 1710. doi 10.1134/1.1130640

[7] Panyaev I.S., Sannikov D.G. // J. Optical Society of America B. 2016. V. 33. P. 220. doi 10.1364/JOSAB. 33.000220

[8] Агравал Г.П. // Нелинейная волоконная оптика. М.: Мир, 1996. 323 c.; Agrawal G.P. Nonlinear Fiber Optics. N. Y: Academic Press, 1995. 592 p. doi 10.1016/C2009-0-21165-2

[9] Беспрозванных В.Г., Первадчук В.П. // Нелинейные эффекты в волоконной оптике. Пермь: Изд-во Перм. нац. исслед. политехн. ун-та, 2011. 228 с.

[10] Kip D. // Appl. Phys. B: Lasers A. Optics. 1998. V. 67. P. 131.

[11] Петров М.П., Степанов С.И., Хоменко А.В. // Фоторефрактивные кристаллы в когерентной оптике / отв. ред. В.В. Брыксин. СПб.: Наука, 1992. 317 с.; Petrov M., Stepanov S., Khomenko A. // Photorefractive Crystals in Coherent Optics, Berlin: Springer-Verlag, 1991. doi 10.1007/978-3-540-47056-4
[12] Виноградов А.П., Ерохин С.Г., Грановский А.Б., Инуе М. // Радиотехн. и электрон. 2004. Т. 49. С. 726.

[13] Shadrivov I.V., Sukhorukov A.A., Kivshar Yu.S., Zharov A.A., Boardman A.D., Egan P. // Phys. Rev. E. 2004. V. 69. P. 016617-1. doi 10.1103/PhysRevE.69.016617

[14] Shadrivov I.V., Sukhorukov A.A., Kivshar Yu.S. // Phys. Rev. E. 2003. V. 67. P. 057602. doi 10.1103/PhysRevE.67.057602

[15] Kivshar Yu.S., Kosevich A.M., Chubykalo O.A. // Phys. Rev. A. 1990. V. 41. P. 1677. doi 10.1103/PhysRevA.41.1677

[16] Abdullaev F.Kh., Baizakov B.B., Umarov B.A. // Opt. Commun. 1998. V. 156. P. 341.

[17] Савотченко C.E. // Изв. вузов. Физика. 2004. Т. 47. С. 79; Savotchenko S.E. Russian Phys. J. 2004. V. 47. P. 556. doi 10.1023/B:RUPJ.0000046330.92744.73

[18] Savotchenko S.E. // Modern Phys. Lett. B. 2018. V. 32. N 10. P. 1850120. doi 10.1142/S0217984918501208

[19] Савотченко С.Е. // ЖЭТФ. 2018. Т. 154. № 3(9). С. 514. doi 10.1134/S0044451018090067; Savotchenko S.E. // JETP. 2018. V. 127. N 3 (9). P. 437. doi 10.1134/S1063776118090108

[20] Boardman A.D., Shabat M.M., Wallis R.F. // J. Phys. D: Apll. Phys. 1991. V. 24. P. 1702. doi 10.1088/0022-3727/24/10/002

[21] Савотченко C.E. // Конденсированные среды и межфазные границы. 2017. Т. 19. № 4. С. 567. doi $10.17308 / \mathrm{kcmf} .2017 .19 / 238$

[22] Савотченко C.E. // Вестн. Воронежского гос. ун-та. Сер. Физика. Математика. 2018. № 1. С. 44.

[23] Savotchenko S.E. // Surfaces and Interface. 2018. V. 13. P. 157. doi 10.1016/j.surfin.2018.09.008

[24] Zhang T.H., Ren X.K., Wang B.H., Lou C.B., Hu Z.J., Shao W.W., Xu Y.H., Kang H.Z., Yang J., Yang D.P., Feng L., Xu J.J. // Phys. Rev. A. 2007. V. 76. 013827. doi 10.1103/PhysRevA.76.013827

[25] Усиевич Б.А., Нурлигареев Д.Х., Сычугов В.А., Ивлева Л.И., Лыков П.А., Богодаев Н.В. // Квант. электрон. 2010. Т. $40 . \quad$ № $\quad$ 5. Usievich B.A., Nurligareev D.Kh., Sychugov V.A., Ivleva L.I., Lykov P.A., Bogodaev N.V. // Quantum Electronics. 2010. V. 40. P. 437. doi 10.1070/QE2010v040n05ABEH014223

[26] Нурлигареев Д.Х., Усиевич Б.А., Сычугов В.А., Ивлева Л.И. // Квант. электрон. 2013. Т. 43. С. 14; Nurligareev D.Kh., Usievich B.A., Sychugov V.A., Ivleva L.I. // Quantum Electronics. 2013. V. 43. P. 14. doi 10.1070/QE2013v043n01ABEH014913

[27] Четкин С.А., Ахмеджсанов И.М. // Квант. электрон. 2011. Т. 41. С. 980; Chetkin S.A., Akhmedzhanov I.M. // Quantum Electronics. 2011. V. 41. P. 980. doi 10.1070/QE2011v041n11ABEH014660

[28] Mikhalake D., Fedyanin V.K. // Theor. Mah. Phys. 1983. V. 54. P. 443.

[29] Герасимчук И.В., Ковалев А.С. // ФНТ. 2000. Т. 26. С. 799; Gerasimchuk I.V., Kovalev A.S. // Low Temp. Phys. 2000. V. 26. P. 586. doi 10.1063/1.1289129

[30] Hamada M.S., Assa'd A.I., Ashour H.S., Shabat M.M. // J. Microwaves and Optoelectr. 2006. V. 5. P. 45.

[31] Hamada M.S., Assa'd A.I. // J. Al Azhar University-Gaza (Natural Sciences). 2011. V. 13. P. 93.

[32] Assa'd A.I., Hamada M.S. // Turk. J. Phys. 2012. V. 36. P. 207. doi 10.3906/fiz-1106-8

[33] Коровай О.В., Хаджи П.И. // ФТТ. 2008. Т. 50. С. 1116; Korovai O.V., Khadzhi P.I. // Phys. Solid State. 2008. V. 50. P. 1165. doi 10.1134/S1063783408060279 
[34] Коровай О.В., Хаджи П.И. // ФТТ. 2010. Т. 52. С. 2277; Korovai O.V., Khadzhi P.I. // Phys. Solid State. 2010. V. 52. P. 243. doi $10.1134 / \mathrm{S} 106378341$

[35] Савотченко С.E. // Нелинейный мир. 2018. Т. 3. С. 25.

[36] Савотченко C.E. // ФТТ. 2019. Т. 61. С. 571. doi 10.21883/FTT.2019.04.47403.147; Savotchenko S.E. // Phys. Solid State. 2019. V. 61. N 4. P. 495. doi 10.1134/S1063783419040255

[37] Савотченко C.E. // ФТТ. 2019. Т. 61. C. 698. doi 10.21883/FTT.2019.04.47415.32; Savotchenko S.E. // Phys. Solid State. 2019. V. 61. N 4. P. 575. doi 10.1134/S1063783419040243

[38] Duree G.C., Shultz J.L., Salamo G.J., Segev M., Yariv A., Crossignani B., Porto P., Sharp E.J., Neurgaonkar R.R. // Phys. Rev. Lett. 1993. V. 71. N 4. P. 533-536.

[39] Castillo M.D.I., Aguilar P.A.M., Sanches-Mondragon J.J., Stepanov S., Vysloukh V. // Appl. Phys. Lett. 1994. V. 4. P. 408-410.

[40] Zhang D., Li Z., Hu W., Cheng B. // Appl. Phys. Lett. 1995. V. 67. P. 2431. doi 10.1063/1.114597

[41] Fazio E., Renzi F., Rinaldi R., Bertolotti M., Chauvet M., Ramadan W., Petris A., Vlad V.I. // Appl. Phys. Lett. 2004. V. 85. P. 2193. doi 10.1063/1.1794854

[42] Petersen P.M., Marrakchi A., Buchhave P., Andersen P.E. // Ferroelectics. 1995. V. 174. P. 149. doi 10.1080/00150199508216944

[43] Canoglu E., Yang C.M., Garmire E. // Appl. Phys. Lett. 1996. V. 69. P. 316. doi 10.1063/1.118045

[44] Jensen S.J. // Spatial Structures and Temporal Dynamics in Photorefractive Nonlinear Systems. Roskilde, Denmark, 1999. $115 \mathrm{p}$.

[45] Buse K., Denz C., Krolikowski W. // Appl. Phys. B. 2009. V. 95. P. 389. doi 10.1007/s00340-009-3530-z

[46] Naim Ben Ali // Chinese J. Phys. 2017. V. 55. P. 2384. doi 10.1016/j.cjph.2017.10.008

[47] Zhong N., Wang Z., Chen M., Xin X., Wu R., Cen Y., Li Y. // Sensors and Actuators B: Chem. 2018. V. 254. P. 133. doi.org/10.1016/j.snb.2017.07.032 